\title{
Efeitos do extrato aquoso de Ipomoea carnea subsp. fistulosa sobre o desenvolvimento de larvas de Chrysomya albiceps (Wiedemann, 1819) (Calliphoridae: Diptera)
}

\author{
Olimpio Francisco Santos da Cruz Junior ${ }^{1}$ \\ José de Jesus Corrêa-Neto ${ }^{2 *}$ \\ Leonardo Gomes ${ }^{1}$ \\ ${ }^{1}$ Universidade Federal do Pará, Campus Universitário do Marajó-Soure \\ CEP 68.870-000, Soure - PA, Brasil \\ ${ }^{2}$ Instituto Nacional de Pesquisas da Amazônia, Coordenação de Biodiversidade \\ Programa de Pós-Graduação em Entomologia \\ Avenida André Araújo, 2936, CEP 69.067-375. Manaus - AM, Brasil \\ * Autor para correspondência \\ netoentomo@gmail.com
}

Submetido em 20/08/2019

Aceito para publicação em 05/12/2019

\section{Resumo}

Plantas do gênero Ipomoea são conhecidas por ocasionarem intoxicações letais a animais de produção. Porém, pouco se sabe sobre os efeitos de suas toxinas em invertebrados de importância médico-veterinária e forense. Esta pesquisa visou analisar o efeito do extrato aquoso em diferentes concentrações de Ipomoea carnea subsp. fistulosa sobre o tempo de desenvolvimento larval e corporal de imaturos de Chrysomya albiceps (Wiedemann, 1819), uma espécie de importância forense no Brasil. Larvas L1 de C. albiceps foram submetidas a três tratamentos: controle (apenas carne moída), $40 \mathrm{~mL}$ (40 mL de extrato de $I$. carnea subsp. fistulosa para $80 \mathrm{~g}$ de carne moída) e $80 \mathrm{~mL}$ ( $80 \mathrm{~mL}$ de extrato de I. carnea subsp. fistulosa para $80 \mathrm{~g}$ de carne moída). Os tempos de desenvolvimento larval até a fase de pupa foram estatisticamente maiores nos tratamentos de 40 e 80 $\mathrm{mL}$, respectivamente, em relação ao controle. Foi observada também diferença significativa no comprimento total das larvas do controle com os demais tratamentos. Os resultados indicam que o contato com as toxinas presentes em I. carnea subsp. fistulosa retarda o desenvolvimento de larvas de C. albiceps.

Palavras chave: Entomologia forense; Moscas-varejeiras; Plantas tóxicas

\section{Abstract}

Effects of aqueous extracts of Ipomoea carnea subsp. fistulosa on the development of Chrysomya albiceps larvae (Wiedemann, 1819) (Calliphoridae: Diptera). Plants of the genus Ipomoea are known to cause lethal poisoning in farm animals. However, little is known about the effects of their toxins on veterinary and forensic invertebrates. This study aimed to determine the effect of an aqueous extract of Ipomoea carnea subsp. fistulosa at different concentrations on larval development time and size of immature Chrysomya albiceps (Wiedemann, 1819), a species of forensic importance in Brazil. C. albiceps L1 larvae were subjected to three treatments: control (ground beef only), $80 \mathrm{~g}$ ground beef mixed with $40 \mathrm{~mL}$ of plant extract) and $80 \mathrm{~g}$ of ground 
beef mixed with $80 \mathrm{~mL}$ of plant extract. Larval development time until the pupal phase was statistically longer in the 40 and $80 \mathrm{~mL}$ extract treatments in relation to the control. The total length of the control larvae was significantly longer than with the extract treatments. The results suggest that the toxins present in I. carnea subsp. fistulosa slow the development of $C$. albiceps larvae.

Key words: Blowflies; Forensic Entomology; Toxic plants

\section{Introdução}

Plantas do gênero Ipomoea, pertencentes à família Convolvulaceae, são nativas da América tropical, com ocorrência nas regiões da América do Sul e Central. No Brasil, a ocorrência desse gênero vai da Amazônia e em todo o litoral da região Norte até os estados do Rio de Janeiro, São Paulo e região Nordeste (KISSMANN; GROTH, 1992). As espécies podem ser encontradas às margens de rios, lagoas, praias marítimas, em terrenos abandonados e nas margens das estradas (TOKARNIA et al., 2000).

Entre as espécies de Ipomoea que ocorrem no Brasil, I. carnea subsp. fistulosa, conhecida popularmente como algodão bravo, canudo, manjorana, capa-bode ou mata-cabra, é uma planta que causa doença de depósito lisossomal em ruminantes, principalmente em caprinos (TOKARNIA et al., 1960; ARMIÉN et al., 2007; GUEDES et al., 2007) e em outros animais de produção (ARAÚJO et al., 2008; TORTELLI et al., 2008; CARVALHO et al., 2014). O princípio ativo de I. carnea subsp. fistulosa é o alcaloide indolizidínico swainsonina, que restringe a eficiência enzimática das enzimas $\alpha$-manosidase lisossomal e $\alpha$-manosidase II do aparelho de Golgi, prejudicando a produção de oligossacarídeos complexos e levando a quadros clínicos severos de intoxicações.

A intoxicação efetiva dessa planta depende da quantidade de ingestão de suas folhas e galhos, nos quais existe uma maior concentração de swainsonina, substância presente nessas plantas e que atua no sistema nervoso central (TOKARNIA et al., 1960; ARMIÉN et al., 2007). Os efeitos de I. carnea subsp fistulosa sobre larvas de insetos ainda são desconhecidos. Entretanto, devido ao seu efeito tóxico sobre a atividade lisossomal de vertebrados ser bem descrito na literatura científica e ao fato de que essa organela celular está presente também em invertebrados, pode se pressupor que exista algum tipo de efeito deletério sobre as células dos invertebrados expostas a compostos oriundos da planta. Uma área em expansão no Brasil é a Entomologia Forense, que estuda processos biológicos e ecológicos de insetos e outros invertebrados que usam carcaças de vertebrados para inúmeros aspectos ecológicos como alimentação e reprodução, com o objetivo de determinar o intervalo pós-morte (IPM) (CORREAA-NETO; GOMES, 2018).

Novas técnicas, dentro da entomologia forense, estão sendo testadas e tem revelado novas opções para responder questões periciais. Uma dessas alternativas é a entomotoxicologia, que detecta a presença de drogas, toxinas e os efeitos destas sobre o desenvolvimento de imaturos de Diptera e Coleoptera em cadáveres (THYSSEN; GRELLA, 2011).

Até o momento, tem se testado os efeitos de drogas ilícitas, medicamentos e extratos vegetais sobre o desenvolvimento de larvas de moscas necrófagas e de outros grupos de moscas causadoras de problemas na agricultura e pecuária (DELEITO; BORJA, 2008; LIMA, 2009; THYSSEN; GRELLA, 2011; ROHDE et al., 2013; SILVA et al., 2016). Porém, não há estudos testando os efeitos de extratos aquosos de plantas tóxicas sobre o desenvolvimento de insetos imaturos de Calliphoridae.

Chrysomya albiceps (Wiedemann, 1819) é uma das espécies mais abundantes de Calliphoridade no território nacional e a escolha desta espécie como modelo biológico de estudo está relacionada à sua grande abundância na área de estudo (CORRÊA-NETO; GOMES, 2018) e por ser um dos principais insetos de importância forense do Brasil (OLIVEIRA-COSTA, 2011).

Diante do exposto, este estudo teve como objetivo analisar os efeitos do extrato aquoso de I. carnea subsp. fistulosa sobre o desenvolvimento de dípteros imaturos 
de C. albiceps e assinalar o possível uso dessa planta no controle de miíases de outras moscas de importância médica veterinária no Brasil.

\section{Material e Métodos}

\section{Coleta e criação de Chrysomya albiceps}

Os espécimes de C. albiceps foram coletados no terreno da Universidade Federal do Pará, campus de Soure (UFPA - Soure), localizado no município de Soure, Ilha de Marajó, Pará, Brasil. Para captura das moscas, foram utilizadas cinco armadilhas de fabricação caseira segundo o modelo proposto por Amat (2010). Utilizou-se como atrativo para as moscas em cada armadilha $50 \mathrm{~g}$ de carne moída já em decomposição misturada com água filtrada para evitar o ressecamento.

Após a coleta, os exemplares foram submetidos a baixas temperaturas $\left(-20^{\circ} \mathrm{C}\right.$ durante 40 segundos $)$ para que o metabolismo dos espécimes reduzisse sua atividade e fosse possível manuseá-los, para que ocorresse de forma tranquila a triagem e identificação dos espécimes que foram usados no estudo. Após a triagem e identificação dos exemplares, eles foram transferidos para gaiolas com as dimensões $(30 \times 30 \times 50 \mathrm{~cm})$ contendo uma tela protetora, onde foram alimentados com solução açucarada e água para estimular o folículo ovariano das fêmeas. As gaiolas contendo as moscas ficaram em um galpão protegidas da chuva e de forte exposição solar, porém ficaram expostas a todas as fotofases e variações climáticas diárias.

\section{Preparação dos extratos aquosos}

Para a preparação dos extratos aquosos, os exemplares de I. carnea subsp. fistulosa foram coletados em diferentes localidades do município de Soure, Pará, Brasil para evitar pseudoréplicas filogenéticas. Os exemplares de I. carnea subsp. fistulosa foram identificados de acordo com a chave dicotômica de Ferreira e Miotto (2009). Os extratos foram preparados com os vegetais frescos. Foram utilizadas somente as folhas, estas foram colhidas e trituradas em processador elétrico (Liquidificador) com água destilada na proporção de $20 \% \mathrm{p} / \mathrm{v}$ (peso do vegetal fresco antes da trituração/ volume de água) e deixadas em repouso durante $24 \mathrm{~h}$, em uma sala climatizada $\left(25 \pm 2^{\circ} \mathrm{C}\right)$.

\section{Preparação dos grupos experimentais}

Uma porção de $50 \mathrm{~g}$ de carne bovina moída crua foi ofertada em um recipiente às fêmeas de $C$. albiceps com o objetivo de estimular a oviposição. Os ovos depositados pelas fêmeas foram mantidos sobre o substrato até que as larvas emergissem, permitindo, assim, iniciar a montagem dos grupos experimentais. Foram montados dois grupos experimentais, que ficaram no mesmo galpão mencionado anteriormente, com as seguintes concentrações do extrato testado: $40 \mathrm{~mL}$ de extrato para $80 \mathrm{~g}$ de carne moída e $80 \mathrm{~mL}$ de extrato para $80 \mathrm{~g}$ de carne bovina moída. Simultaneamente foi mantido um grupo controle com $80 \mathrm{~g}$ de carne bovina moída sem adição do extrato de planta tóxica, para avaliar o efeito dos tratamentos experimentais. Para cada grupo foram feitas três réplicas simultaneamente, em cada réplica foram utilizadas 40 larvas recém eclodidas L1, que foram acompanhadas a cada $12 \mathrm{~h}$ até o total de 96 h ou enquanto fosse possível encontrar imaturos vivos. É importante ressaltar que a carne bovina contendo o extrato não foi trocada até o final do experimento. Para obtenção do comprimento total das larvas (CT) foram coletadas dez larvas (L2) aleatoriamente a cada $12 \mathrm{~h}$ e depois devolvidas aos substratos. Para realizar as medidas de comprimento total, foi utilizada uma régua milimétrica.

\section{Análise de dados}

Para avaliar os efeitos dos tratamentos sobre a variável comprimento total, o (CT) de larvas $C$. albiceps, foi aplicado o teste estatístico Anova-one way, sendo testados os pressupostos de Shapiro-Wilk e homecadasticidade através do teste de Levene (ZAR, 2010). Foi aplicado o teste de Tukey para resultados significativos por meio do programa Statistica 7.0. E para avaliar a variável tempo de metamorfose das larvas foi empregada análise estatística descritiva. Os valores considerados significativos foram estabelecidos o valor $\mathrm{p}=<0,05$. 


\section{Resultados}

As larvas submetidas ao tratamento controle apresentaram um padrão de desenvolvimento esperado para a espécie em estudo, começando a empupar na nonagésima sexta hora de experimento (Figura 1). Nos demais tratamentos as larvas demoraram em média $30 \mathrm{~h}$ a mais do que as larvas do grupo controle para dar início ao processo de pupariação (Figura 1).
Foi constatada diferença significativa do variável comprimento total das larvas entre os tratamentos analisados $(\mathrm{F}$-test $(2,09)=21,386 ; \mathrm{p}=0,00)$. As larvas do grupo controle foram em média $0,16 \mathrm{~mm}$ maiores do que as larvas do grupo tratamento que recebeu $40 \mathrm{~mL}$ de solução segundo o Teste de Tukey $(=0,000)$ e $0,18 \mathrm{~mm}$ maiores que as larvas do grupo tratamento que recebeu $80 \mathrm{~mL}$ de solução segundo o Teste de Tukey $(=0,000)$ (Figura 2).

FIGURA 1: Tempo de desenvolvimento de larvas L2 a L3 de Chrysomya albiceps submetidas a uma dieta de carne bovina contendo 40 $\mathrm{mL}$ e $80 \mathrm{~mL}$ de extrato aquoso de Ipomoea carnea subsp. fistulosa.

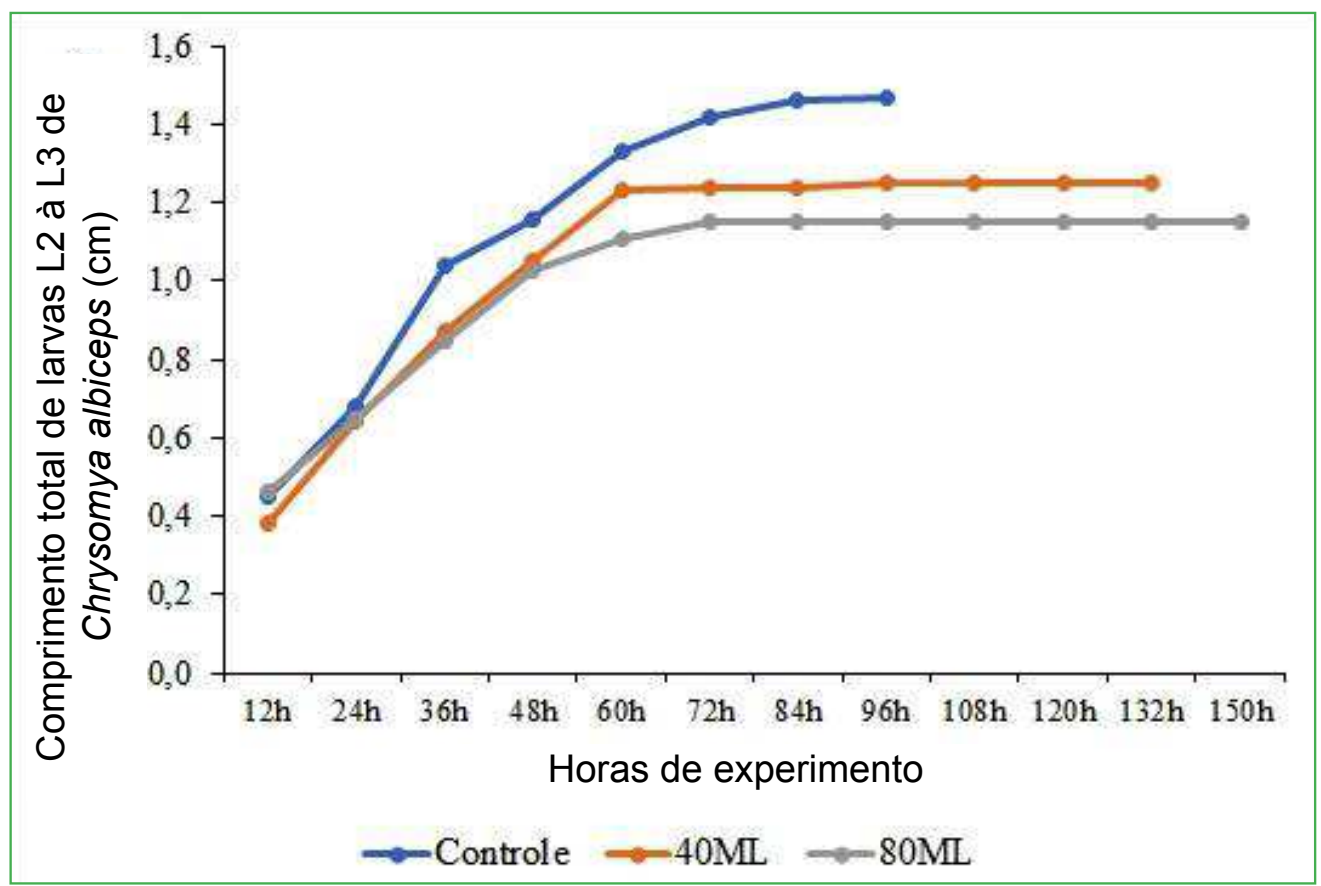


FIGURA 2: Comprimento total de larvas L2 a L3 de Chrysomya albiceps submetidas a uma dieta de carne bovina com $40 \mathrm{~mL}$ e $80 \mathrm{~mL}$ de extrato aquoso de Ipomoea carnea subsp. fistulosa. As barras de erros representam o erro padrão com um intervalo de confiança de $95 \%$. Letras diferentes acima das barras indicam as diferenças entre as médias pelo teste de Tukey.

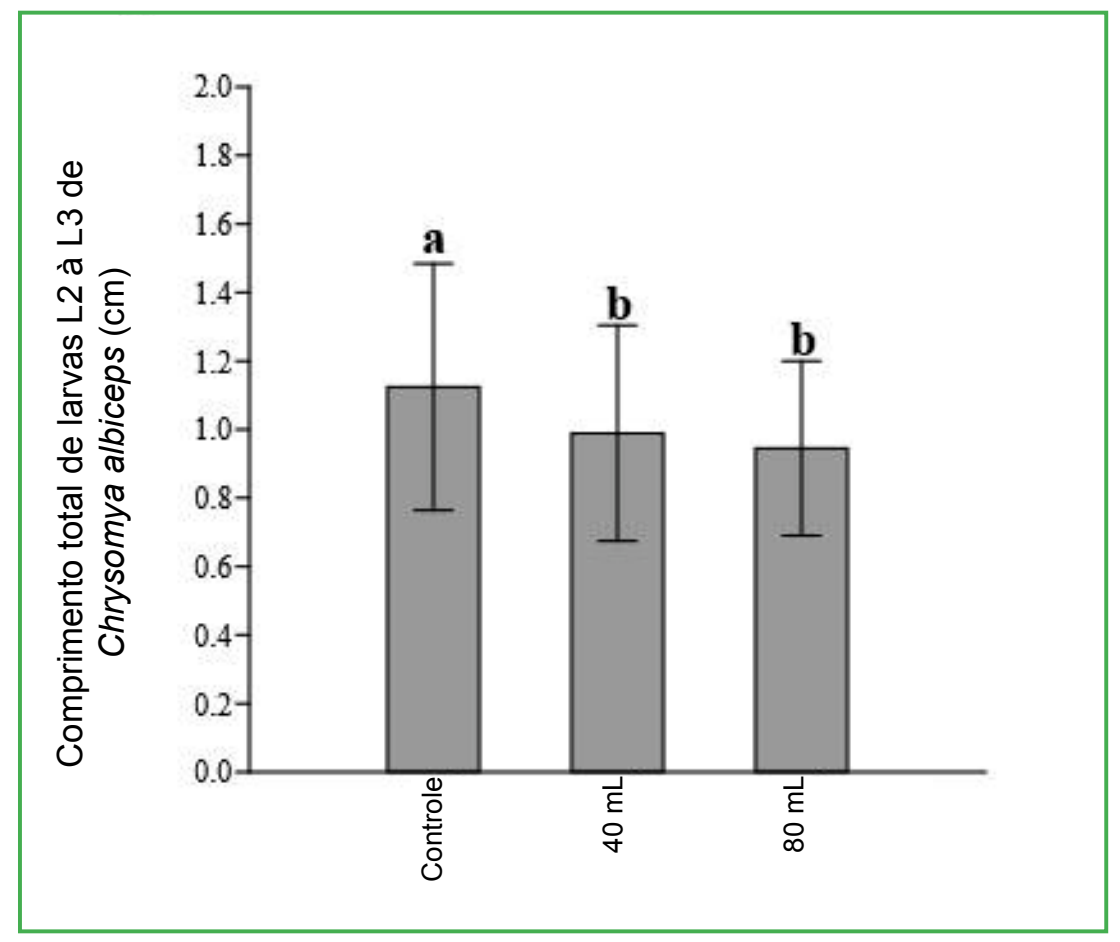

\section{Discussão}

Foi observado que as larvas do grupo controle seguiram um padrão de tempo larval já esperado para a espécie em estudo, chegando ao estágio de pupa às 96 h (ESTRADA et al., 2009). Já os tratamentos que receberam 40 e $80 \mathrm{~mL}$ da solução testada seguiram um padrão incomum, pois houve um retardo no desenvolvimento até o estágio de pupa. Esse aumento no tempo larval da espécie em estudo pode estar atribuído à presença de substâncias tóxicas existentes nesses extratos.

Segundo Haraguchi et al. (2003), espécies de Ipomoea possuem, em sua composição química, swainsonina, um alcaloide indolizidínico ativo, que causa inúmeras modificações no comportamento e nos aspectos físicos de animais vertebrados, como falta de apetite, perda de peso, sonolência e excitabilidade, inibindo assim o ritmo de crescimento (SCHWARZ et al., 2004; LIMA et al., 2013; AMBIGA; JEYARAJ, 2015), corroborando os nossos achados.
Em relação ao comprimento total, as larvas do grupo controle tiveram os maiores comprimentos em relação às larvas submetidas nos tratamentos de $40 \mathrm{e}$ $80 \mathrm{~mL}$ de extrato vegetal. Esses resultados podem ser explicados pela presença de componentes moleculares nessas plantas, que atuam como inibidores de funções enzimáticas que são responsáveis pela metabolização dos carboidratos, impossibilitando a absorção de nutrientes necessários para o processo de desenvolvimento (SCHWARZ et al., 2004).

Além disso, as manifestações clínicas observadas nas larvas, como falta de atividade, desinteresse em consumir o substrato e excitabilidade em comparação ao grupo controle, podem ter contribuído com a falta de ingestão do alimento, pois quanto menos alimento ingerido, mais lento será o desenvolvimento do indivíduo. No ramo da entomotoxicologia, substâncias tóxicas presentes em substratos que servem de alimento para inúmeros insetos necrófagos podem trazer consequências no desenvolvimento e na taxa de mortalidade desses indivíduos e podem induzir a erros 
na estimativa do IPM em procedimentos criminais (INTRONA et al., 2001; WOLF et al., 2006; ESTRADA et al., 2009; RAMOS-PASTANA; WOLFF, 2017).

Nossos resultados também podem servir para subsidiar pesquisas no contexto do controle biológico, tema que vem crescendo nos últimos anos. Embora os tratamentos não tenham levado à morte das larvas ao final do experimento, houve um retardo em todo o processo de desenvolvimento desses insetos. Esse dado pode sugerir a hipótese de que com maiores concentrações de extratos aquosos de I. carnea subsp. fistulosa, possivelmente as larvas não chegariam ao processo final da metamorfose e morreriam (CHAABAN et al., 2018; 2019; SEUGLING et al., 2019).

Contudo, concluímos que extratos aquosos de $I$. carnea subsp. fistulosa podem ser inibidores do processo de desenvolvimento de larvas de C. albiceps, podendo levar a falsas estimativas do IPM em periciais criminais. Além disso, com esses resultados é possível inferir que as substâncias oriundas de I. carnea subsp. fistulosa podem ser eficientes no controle de imaturos de espécies causadoras de miíases e transmissoras de patógenos para o ser humano.

\section{Agradecimentos}

À Universidade Federal do Pará, Campus Soure, por toda a infraestrutura que nos disponibilizou para a execução deste estudo. Aos avaliadores do artigo que fizeram excelentes considerações que melhoraram de forma significativa este artigo.

\section{Referências}

AMAT, E. Notes on necrophagous flies (Diptera: Calyptratae) associated to fish carrion in Colombian Amazon. Acta Amazonica, Manaus, v. 40, n. 2, p. 397-400, 2010.

AMBIGA, S.; JEYARAJ, M. Evaluation of in vitro antioxidant activity of Ipomoea carnea Jacq. International Journal of Current Microbiology and Applied Sciences, Tamilnadu, v. 4, n. 5, p. 327-338, 2015.

ARAÚJO, J. A. S.; CORRÊA, F. R.; MEDEIROS, R. M. T.; SOARES, M. P.; OLIVEIRA, D. M.; CARVALHO, F. K. L.; Intoxicação experimental por Ipomoea asarifolia (Convolvulaceae) em caprinos e ovinos. Pesquisa Veterinária Brasileira, Rio de Janeiro, v. 28, n. 10, p. 488-494, 2008.
ARMIÉN, A. G.; TOKARNIA, C. H.; PEIXOTO, P. V.; FRESE, K. Spontaneous and experimental glycoprotein storage disease of goats induced by Ipomoea carnea subsp. fistulosa (Convolvulaceae). Veterinary Pathology, Basel, v. 44, p. 170-184, 2007.

CARVALHO, F. K. L.; DANTAS, A. F. M.; CORRÊA, F. R.; PIRES, J. P. S.; SILVA, F. O. R. Intoxicação por Ipomoea asarifolia em bovinos e ovinos no Rio Grande do Norte. Pesquisa Veterinária Brasileira, Rio de Janeiro, v. 34, n. 11, p. 1073-1076, 2014.

CHAABAN, A.; MARTINS, C. E. N.; BRETANHA, L. C.; MICKE, G. A.; CARRER, A. R., ROSA, N. F.; FERREIRA, L.; MOLENTO, M. B. Insecticide activity of Baccharis dracunculifolia essential oil against Cochliomyia macellaria (Diptera: Calliphoridae). Natural Product Research, Roma, v. 32, p. 2954-2958, 2018.

CHAABAN, A.; RICHARDI, V. S.; CARRER, A. R.; BRUM, J. S.; CIPRIANO, R. R.; MARTINS, C. E. N.; SILVA, M. A. N.; DESCHAMPS, C.; MOLENTO, M. B. Insecticide activity of Curcuma longa (leaves) essential oil and its major compound $\alpha$-phellandrene against Lucilia cuprina larvae (Diptera: Calliphoridae): Histological and ultrastructural biomarkers assessment. Pesticide Biochemistry and Physiology, Massachusetts, v. 153, p. 17-27, 2019.

CORRÊA-NETO, C. J. J.; GOMES, L. Abundância e flutuação populacional de Chrysomya albiceps (Wiedmam, 1819) (Diptera: Calliphoridae) associadas a carcaças de Sus scrofa (Linnaeus, 1758) na região norte do Brasil. Revista Brasileira de Zoociências, Juiz de Fora, v. 19, n. 1, p. 67-76, 2018.

DELEITO, R. S. C.; BORJA, M. E. G. Nim (Azadirachta indica): uma alternativa no controle de moscas na pecuária. Pesquisa Veterinária Brasileira. Rio de Janeiro, v. 28, n. 6, p. 293-298, 2008.

ESTRADA, D. A.; GRELLA, M. D.; THYSSEN, P. J.; LINHARES, A. X. Taxa de desenvolvimento de Chrysomya albiceps (Wiedemann) (Diptera: Calliphoridae) em dieta artificial acrescida de tecido animal para uso forense. Neotropical Entomology, Londrina, v. 38, n. 2, p. 203-207, 2009.

FERREIRA, A. P. P.; MIOTTO, S. T. S. Sinopse das espécies de Ipomoea L. (Convolvulaceae) ocorrentes no Rio Grande do Sul, Brasil. Revista Brasileira de Biociências, Porto Alegre, v. 7, n. 4, p. 440-453, 2009.

GUEDES, K. M. R.; RIET-CORREA, F.; DANTAS, A. F. M.; SIMÕES, S. V. D.; MIRANDA-NETO, E. G.; NOBRE, V. T. M.; MEDEIROS, R. M. T. Doenças do sistema nervoso central de caprinos e ovinos no semi-árido. Pesquisa Veterinária Brasileira, Rio de Janeiro, v. 27, p. 25-34, 2007

HARAGUCHI, M.; GORNIAK, S. L.; IKEDA, K.; MINAMI, Y.; KATO, A.; WATSON, A. A.; NASH, R. J.; MOLYNEUX, R. J.; ASANO, N. Alkaloidal components in the poisonous plant, Ipomoea carnea (Convolvulaceae). Journal of Agricultural and Food Chemistry, Washington, v. 51, p. 4995-5000, 2003.

INTRONA, F.; CAMPOBASSO, C. P.; GOFF, M. L. Entomotoxicology. Forensic Science International, Lausanne, v. 120, p. 42-47, 2001.

KISSMANN, K. G.; GROTH, D. Plantas infestantes e nocivas. São Paulo: Basf Brasileira, 1992. 789 p.

LIMA, B. M. F. V.; MOREIRA, J. O. T.; ARAGÃO, C. A. Avaliação de extratos vegetais no controle de mosca-branca, Bemisia tabaci 
biótipo B em abóbora. Revista Ciência Agronômica, Fortaleza, v. 44, n. 3, p. 622-627, 2013.

LIMA, P. G. C. Detecção e estudo sobre o efeito da metanfetamina e do ecstasy no desenvolvimento de imaturos de três espécies de Chrysomya (Diptera: Calliphoridae) de importância forense. 2009. 79 f. Dissertação (Mestrado em Biologia Geral e Aplicada) Universidade Estadual Paulista, Botucatu. 2009.

OLIVEIRA-COSTA, J. Entomologia forense: quando os insetos são os vestígios. 3. ed. Campinas: Millennium, 2011. 520 p.

RAMOS-PASTANA, Y.; WOLFF, M. Posmortem interval estimation based on Chrysomya albiceps (Diptera, Calliphoridae) in a forensic case in the Andean Amazon, Caquetá, Colombia. Acta Amazonica, Manaus, v. 47, n. 4, p. 369-374, 2017.

ROHDE, C.; JÚNIOR, M. A.; SILVA, K. P.; RAMALHO, O. R. K. Efeitos de estratos vegetais aquosos sobre a mosca-dasfrutas Ceratitis capitata (Wiedemann) (Diptera: Tephritidae). Agricultural Entomology, São Paulo, v. 80, n. 4, p. 407-415, 2013. SCHWARZ, A.; HOSOMI, R. Z.; HENRIQUEL, B. S.; HUESA, I.; GARDNER, D.; HARAGUCHI, M.; GORNIAK, S. L.; BERNARDI, M. M.; SPINOSA, H. S. Identificação de princípios ativos presentes na Ipomoea carnea brasileira. Brazilian Journal of Pharmaceutical Sciences, São Paulo, v. 40, n. 2, p. 181-187, 2004.

SEUGLING, J.; KUHNEN, S.; PEREIRA DE BARROS, G.; VELERINHO, M. B.; MAZZARINO, L.; BRICARELLO, P. A. Development of Baccharis dracunculifolia (Asteraceae) essential oil nanoemulsion and its biological activity on pre-pupae of Cochliomyia hominivorax (Diptera: Calliphoridae). Journal Pharmacy Pharmacology, London, v. 7, p. 293-308, 2019.
SILVA, G. F.; COSTA, M. E.; FERREIRA, R. R.; SILVA, L. E. F.; ARAUJO, L. E. Efeito de diferentes concentrações do extrato aquoso de folhas de nim na mortalidade da mosca minadora Liriomyza sativae Blanchard (Diptera: Agromyzidae). Revista Agroambiente, Rio Branco, v. 10, n. 4, p. 381-396, 2016.

THYSSEN, J. P.; GRELLA, D. M. Efeito da escopolamina sobre o desenvolvimento de Chrysomya putoria (Diptera: Calliphoridae) e sua importância para a estimativa do intervalo pós-morte. Revista Brasileira de Criminalística, Brasília, v. 1, p. 39-42, 2011.

TOKARNIA, C. H.; DÖBEREINER, J.; CANELLA, C. F. C. Estudo experimental sobre a toxidez do "canudo" (Ipomoea fistulosa Mart.) em ruminantes. Arquivos do Instituto de Biologia Animal, São Paulo, v. 3, p. 59-71, 1960.

TOKARNIA, C. H.; DÖBEREINER, J.; PEIXOTO, P. V. Plantas tóxicas do Brasil. Rio de Janeiro: Editora Helianthus, 2000. 310 p. TORTELli, F. P.; BARBOSA, J. D.; OLIVEIRA, C. M. C.; DUARTE, M. D.; CERQUEIRA, V. D.; OLIVEIRA, C. A.; CORRÊA, F. R.; CORRÊA, G. R. Intoxicação por Ipomoea asarifolia em ovinos e bovinos na Ilha de Marajó. Pesquisa Veterinária Brasileira, Rio de Janeiro, v. 28, n. 12, p. 622-626, 2008.

WOLFF, M.; ZAPATA, Y.; MORALES, G.; BENECKE, M. Detección y cuantificación de propoxur em la sucesión de insectos de importância médico-legal. Revista Colombiana de Entomologia, Bogotá, v. 32, n. 2, p. 159-164, 2006.

ZAR, J. H. Biostatistical analysis. New Jersey: Prentice Hall, 2010. 944 p. 